\title{
Data Sensor Fusion for Autonomous Robotics
}

\author{
Özer Çiftçioğlu and Sevil Sariyildiz \\ Delft University of Technology, \\ Faculty of Architecture, Delft \\ The Netherlands
}

\section{Introduction}

Multi-sensory information is a generic concept since such information is of concern in all robotic systems where information processing is central. In such systems for the enhancement of the accurate action information redundant sensors are necessary where not only the number of the sensors but also the resolutional information of the sensors can vary due to information with different sampling time from the sensors. The sampling can be regular with a constant sampling rate as well as irregular. Different sensors can have different merits depending on their individual operating conditions and such diverse information can be a valuable gain for accurate as well as reliable autonomous robot manipulation via its dynamics and kinematics. The challenge in this case is the unification of the common information from various sensors in such a way that the resultant information presents enhanced information for desired action. One might note that, such information unification is a challenge in the sense that the common information is in general in different format and different size with different merits. The different qualities may involve different accuracy of sensors due to various random measurement errors. Autonomous robotics constitutes an important branch of robotics and the autonomous robotics research is widely reported in literature, e.g. (Oriolio, Ulivi et al. 1998; Beetz, Arbuckle et al. 2001; Wang and Liu 2004). In this branch of robotics continuous information from the environment is obtained by sensors and real-time processed. The accurate and reliable information driving the robot is essential for a safe navigation the trajectory of which is in general not prescribed in advance. The reliability of this information is to achieve by means of both physical and analytical redundancy of the sensors. The accuracy is obtained by coordinating the sensory information from the redundant sensors in a multisensor system. This coordination is carried out by combining information from different sensors for an ultimate measurement outcome and this is generally termed as sensor fusion. Since data is the basic elements of the information, sometimes to emphasize this point the fusion process is articulated with data as data fusion where the sensor fusion is thought to be as a synonym. Some examples are as follows.

"Data fusion is the process by which data from a multitude of sensors is used to yield an optimal estimate of a specified state vector pertaining to the observed system." (Richardson and Marsh 1988)

"Data fusion deals with the synergistic combination of information made available by various knowledge sources such as sensors, in order to provide a better understanding of a given scene." (Abidi and Gonzales 1992) 
"The problem of sensor fusion is the problem of combining multiple measurements from sensors into a single measurement of the sensed object or attribute, called the parameter." (McKendall and Mintz 1992; Hsin and Li 2006)

The ultimate aim of information processing as fusion is to enable the system to estimate the state of the environment and in particular we can refer to the state of a robot's environment in the present case. A similar research dealing with this challenge, namely a multiresolutional filter application for spatial information fusion in robot navigation has been reported earlier (Ciftcioglu 2008) where data fusion is carried out using several data sets obtained from wavelet decomposition and not from individual sensors. In contrast with the earlier work, in the present work, in a multi-sensor environment, fusion of sensory information from different sensors is considered. Sensors generally have different characteristics with different merits. For instance a sensor can have a wide frequency range with relatively poor signal to noise ratio or vice versa; the response time of the sensor determines the frequency range. On the other hand sensors can operate synchronized or non-synchronized manner with respect to their sampling intervals to deliver the measurement outcomes. Such concerns can be categorized as matters of sensor management although sensor management is more related to the positioning of the sensors in a measurement system. In the present work data fusion sensor fusion and sensor management issues are commonly are referred to as sensor fusion. The novelty of the research is the enhanced estimation of the spatial sensory information in autonomous robotics by means of multiresolutional levels of information with respect to sampling time intervals of different sensors. Coordination outcome of such redundant information reflects the various merits of these sensors yielding enhanced positioning estimation or estimate the state of the environment. To consider a general case the sensors are operated independently without a common synchronizing sampling command, for instance. The multiresolutional information is obtained from sensors having different resolutions and this multiple information is synergistically combined by means of inverse wavelet transformation developed for this purpose in this work. Although wavelet-based information fusion is used in different applications (Hong 1993; Hsin and Li 2006), its application in robotics is not common in literature. One of the peculiarities of the research is essentially the application of wavelet-based dynamic filtering with the concept of multiresolution as the multiresolution concept is closely tied to the discrete wavelet transform. The multiresolutional dynamic filtering is central to the study together with the Kalman filtering which has desirable features of fusion. Therefore the vector wavelet decomposition is explained in some detail. For the information fusion process extended Kalman filtering is used and it is also explained in some detail emphasizing its central role in the fusion process. In an autonomous robot trajectory the estimation of angular velocity is not a measurable quantity and it has to be estimated from the measurable state variables so that obstacle avoidance problem is taken care of. The angular velocity estimation in real-time is a critical task in autonomous robotics and from this viewpoint, the multiresolutional sensor-based spatial information fusion process by Kalman filtering is particularly desirable for enhanced robot navigation performance. In particular, the multiresolutional sensors provide diversity in the information subject to fusion process. In this way different quality of information with respective merits are synergistically combined.

The motivation of this research is the use of a vision robot for an architectural design and the architectural artifacts therein from the viewpoint of human perception, namely to investigate the perceptual variations in human observation without bias. The similar 
perception centered research by a human can have an inherent bias due to the interests and background of that human. In this respect, a robot can be viewed as an impartial observer with emulated human perception. Therefore, in this research, the sensory information is treated as robot's visual perception as an emulation of that of a human. A theory for the human perception from a viewpoint of perception quantification and computation is presented earlier (Ciftcioglu, Bittermann et al. 2007; Ciftcioglu 2008). The robot can be a physical real artifact autonomously wandering in an architectural environment. Or alternatively, it can be a virtual robot, wandering in a virtual reality environment. Both cases are equally valid utilization options in the realm of perceptual robotics in architecture. Apart from our interest on human perception of architectural artifacts as motivation, the present research is equally of interest to other adjacent robotics research areas like social robots which are closely related to perception robots. Namely, thanks to the advancements in robotics, today the social robots are more and more penetrating in social life as an aid to many human endeavors. With the advent of rapid progresses in robotics and evolutions on hardware and software systems, many advanced social, service and surveillance mobile robots have been coming into realization in the recent decades; see for instance, http:/spectrum.ieee.org/robotics. One of the essential merits of such robots is the ability to detect and track people in the view in real time, for example in a care center. A social robot should be able to keep eye on the persons in the view and keep tracking the persons of concern for probable interaction (Bellotto and Hu 2009). A service robot should be aware of people around and track a person of concern to provide useful services. A surveillance robot can monitor persons in the scene for the identification of probable misbehavior. For such tasks, detecting and tracking multiple persons in often crowded and cluttered scenes in public domain or in a working environment is needed. In all these challenging scenarios perceptual mobile robotics can give substantial contribution for the functionality of such special variety of robots in view of two main aspects. One aspect is vision, which is not the subject-matter of this work. The other aspect is the sensor-data fusion for effective information processing, which is the subject matter of this research where Kalman filtering is the main machinery, as it is a common approach in mobile robotics for optimal information processing.

The further organization of the present work is as follows. After the description of Kalman filtering and wavelet transform in some detail, detailed description of optimal fusion process of information from different multiresolutional levels is presented. The optimality is based on minimum fusion estimation error variance. Finally, autonomous robot implementation is described with the computer experiments the results of which are illustrated by means of both true and estimated trajectories demonstrating the effective multisensor-based, multiresolutional fusion. The work is concluded with a brief discussion and conclusions.

\section{Kalman filter}

\subsection{Description of the system dynamics}

Kalman filtering theory and its applications are well treated in literature (Jazwinski 1970; Gelb 1974; Kailath 1981; Maybeck 1982; Brown 1983; Sorenson 1985; Mendel 1987; Grewal and Andrews 2001; Simon 2006). In order to apply Kalman filtering to a robot movement the system dynamics must be described by a set of differential equations which are in statespace form, in general 


$$
\frac{d x}{d t}=F x+\mathrm{G} u+w
$$

where $x$ is a column vector with the states of the system, $F$ the system dynamics matrix, $u$ is the control vector, and $w$ is a white noise process vector. The process noise matrix $Q$ is related to the process-noise vector according to

$$
Q=\mathrm{E}\left[w w^{\mathrm{T}}\right]
$$

The measurements are linearly related to the states according to

$$
z=H x+v
$$

where $z$ is the measurement vector, $H$ is the measurement matrix, and $v$ is measurement noise vector which is element-wise white. The measurement noise matrix $R$ is related to the measurement noise vector $v$ according to

$$
R=\mathrm{E}\left[v v^{\mathrm{T}}\right]
$$

In discrete form, the Kalman filtering equations become

$$
\begin{aligned}
& x_{k}=\Phi_{k} x_{k-1}+\mathrm{K}_{\mathrm{k}}\left(\mathrm{z}_{\mathrm{k}}-\mathrm{H} \Phi_{k} x_{k-1}-\mathrm{HG}_{\mathrm{k}} u_{\mathrm{k}-1}\right)+\mathrm{G}_{\mathrm{k}} u_{\mathrm{k}-1} \\
& \mathrm{z}_{\mathrm{k}}=H x_{k}+v_{k}
\end{aligned}
$$

where $\Phi_{k}$ system transition matrix, $K_{k}$ represents he Kalman gain matrix and $G_{k}$ is obtained from

$$
G_{k}=\int_{0}^{T} \Phi(\tau) \mathrm{G}(\tau) \mathrm{d} \tau
$$

where $\mathrm{T}$ is the sampling time interval and the computation of $\Phi(\mathrm{t})$ is given shortly afterwards in (13). In this research information processing from the sensors for estimation is concerned. The control signal $(\boldsymbol{u})$ is not involved in the filtering operation. Hence the Kalman filtering equation for this case becomes

$$
x_{k}=\Phi_{k} x_{k-1}+\mathrm{K}_{\mathrm{k}}\left(\mathrm{z}_{\mathrm{k}}-\mathrm{H} \Phi_{k} x_{\mathrm{k}-1}\right)
$$

While the filter is operating, the Kalman gains are computed from the matrix Riccati equations:

$$
\begin{aligned}
& M_{k}=\Phi_{k} P_{k-1} \Phi_{k}^{T}+Q_{k} \\
& K_{k}=M_{k} H^{T}\left(H M_{k} H^{T}+R_{k}\right)^{-1} \\
& P_{k}=\left(I-K_{k} H\right) M_{k}
\end{aligned}
$$

where $P_{k}$ is a covariance matrix representing errors in the state estimates after an update and $M_{k}$ is the covariance matrix representing errors in the state estimates before an update. The discrete process noise matrix $Q_{k}$ can be found from the continuous process-noise matrix $Q$ according to 


$$
Q_{k}=\int_{0}^{T} \Phi(\tau) \mathrm{Q} \Phi^{T}(\tau) \mathrm{d} \tau
$$

where $\Phi(t)$ is given in (13). When robot movement is along a straight line with a constant speed $v_{x}$, the $x$ component of the system dynamic model is given by

$$
x=a_{0}+v_{x} t
$$

It is to note that the angular speed $\omega=0$. The system dynamics in state-space form is given by

$$
\left[\begin{array}{l}
\dot{x} \\
\ddot{x}
\end{array}\right]=\left[\begin{array}{ll}
0 & 1 \\
0 & 0
\end{array}\right]\left[\begin{array}{l}
x \\
\dot{x}
\end{array}\right]
$$

Where the system dynamics matrix $F$ is given by

$$
F=\left[\begin{array}{ll}
0 & 1 \\
0 & 0
\end{array}\right]
$$

The system transition matrix $\Phi$ is computed from inverse Laplace transform of the form

$$
\begin{aligned}
& \Phi(t)=L^{-1}\left\{(s I-F)^{-1}\right\}=e^{F t} \\
& \Phi_{k}=\Phi(t)=\left[\begin{array}{ll}
1 & t \\
0 & 1
\end{array}\right]
\end{aligned}
$$

The discrete fundamental matrix, i.e., system transition matrix can be found from preceding expression by simply replacing time with the sampling time interval of the perception measurements $\mathrm{T}$ or

$$
\Phi_{k}=\Phi(T)=\left[\begin{array}{ll}
1 & T \\
0 & 1
\end{array}\right]
$$

In two dimensional navigation space, i.e., $x y$ plane, the system transition matrix becomes

$$
\Phi_{k}=\left[\begin{array}{llll}
0 & T & 0 & 0 \\
0 & 1 & 0 & 0 \\
0 & 0 & 1 & T \\
0 & 0 & 0 & 1
\end{array}\right]
$$

and the corresponding state vector $X$ is given by

$$
X=\left[\begin{array}{llll}
x & v_{x} & y & v_{y}
\end{array}\right]
$$

In the case of $\omega \neq 0$, i.e., circular movement with an angular velocity, we consider the geometry shown in Figure 1. 


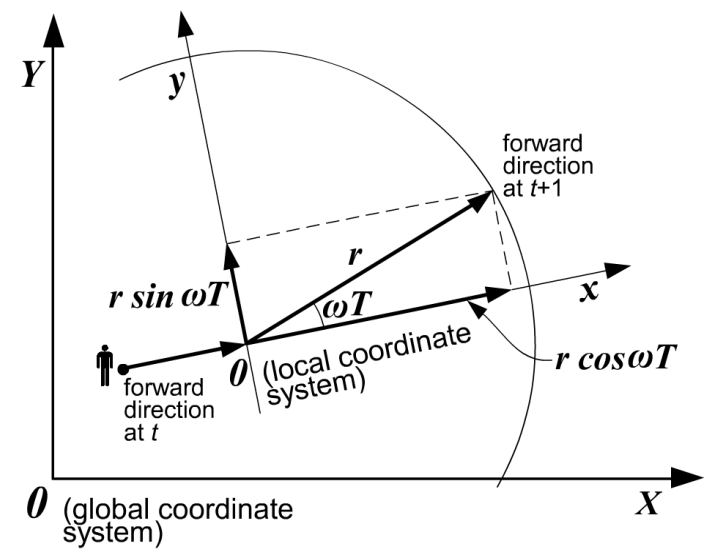

Fig. 1. Geometry with respect to angular deviation during robot navigation

At the local coordinate system, the state variables are

$$
\begin{aligned}
& x_{1}=r \cos (\omega t) \\
& \dot{x}_{1}=-r \omega \sin (\omega t) \\
& x_{2}=r \sin (\omega t) \\
& \dot{x}_{2}=r \omega \cos (\omega t)
\end{aligned}
$$

So that the system dynamics in state-space form in continuous time is given by

$$
\left[\begin{array}{c}
\dot{x_{1}} \\
\ddot{x_{1}} \\
\dot{x_{2}} \\
\ddot{x_{2}}
\end{array}\right]=\left[\begin{array}{llll}
0 & 1 & 0 & 0 \\
0 & 0 & 0 & -\omega \\
0 & 0 & 0 & 1 \\
0 & \omega & 0 & 0
\end{array}\right]\left[\begin{array}{c}
x_{1} \\
\dot{x_{1}} \\
x_{2} \\
\dot{x_{2}}
\end{array}\right]
$$

The system transition matrix $\Phi^{\omega}{ }_{k}$ is computed from inverse Laplace transform of the form

$$
\Phi_{k}^{\omega}(t)=L^{-1}\left\{\left(s I-F_{\omega}\right)^{-1}\right\}=e^{F_{\omega} t}
$$

where $\left(s I-F_{\omega}\right)$ is given by

$$
s I-F_{\omega}=\left[\begin{array}{cccc}
s & -1 & 0 & 0 \\
0 & s & 0 & \omega \\
0 & 0 & s & -1 \\
0 & -\omega & 0 & s
\end{array}\right]
$$

The inverse of (20) yields 


$$
\left[\begin{array}{cccc}
\frac{1}{s} & \frac{1}{s^{2}+\omega^{2}} & 0 & -\frac{\omega}{s\left(s^{2}+\omega^{2}\right)} \\
0 & \frac{1}{s\left(s^{2}+\omega^{2}\right)} & 0 & -\frac{\omega}{s^{2}+\omega^{2}} \\
0 & \frac{\omega}{s\left(s^{2}+\omega^{2}\right)} & \frac{1}{s} & \frac{1}{s^{2}+\omega^{2}} \\
0 & \frac{\omega}{s^{2}+\omega^{2}} & 0 & \frac{1}{s\left(s^{2}+\omega^{2}\right)}
\end{array}\right]
$$

and the inverse Laplace transform of (21) gives the system transition matrix, for $t=T$, as

$$
\Phi_{k}^{\omega}=\left[\begin{array}{cccc}
1 & \frac{\sin (\omega T)}{\omega} & 0 & \frac{\cos (\omega T)-1}{\omega} \\
0 & \cos (\omega T) & 0 & -\sin (\omega T) \\
0 & -\frac{\cos (\omega T)-1}{\omega} & 1 & \frac{\sin (\omega T)}{\omega} \\
0 & \sin (\omega T) & 0 & \cos (\omega T)
\end{array}\right]
$$

During the robot navigation we have obtained two system dynamics models; namely rectilinear straight-ahead and angular rotation cases. To endow the robot to be autonomous the angular velocity should be computed during the navigation. If the perception measurements yield a significant angular velocity, the system dynamics model should switch from linear to non-linear. It is interesting to note that if in (22) $\omega=0$, then it reduces to (15) which is the transition matrix for linear case. In other words, (22) represents inherently the linear case, as well as the rotational robot navigation. If the angular velocity is computed at each step of navigation and if it is non-zero, the robot moves along a non-linear trajectory with each time a deviation $\theta$ from linear trajectory. The linear and non-linear cases are illustrated in figure 2 and figure 3 where the measurements are from sensory visual perception (Ciftcioglu, Bittermann et al. 2007; Ciftcioglu 2008).

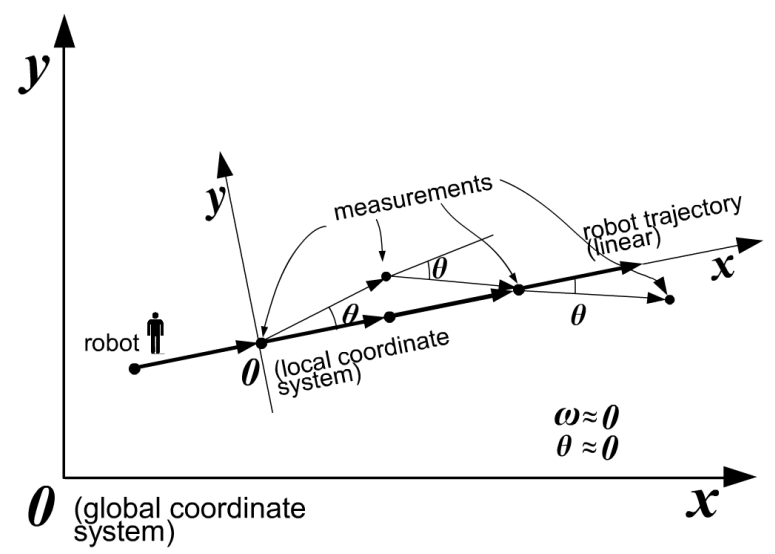

Fig. 2. Measurements along a linear move 


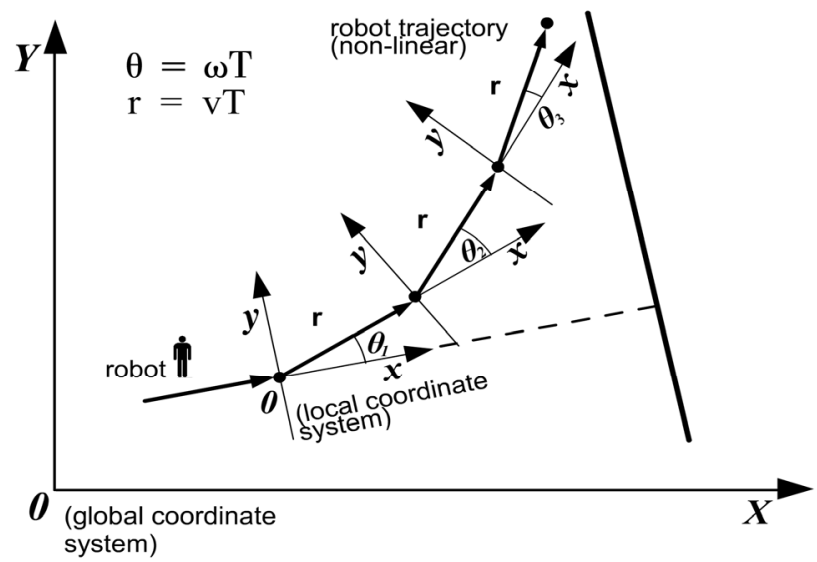

Fig. 3. Robot navigation deviating from a linear move

To compute the angular velocity $\omega$ at each step, it is also selected as a state variable, so that the state variables vector given by (16) is modified to

$$
X^{\omega}=\left[\begin{array}{lllll}
x & v_{x} & y & v_{y} & \omega
\end{array}\right]
$$

However, in this case the system transition matrix in (22) becomes non-linear with respect to $\omega$. In this case Kalman filtering equations should be linearized. This is a form known as extended Kalman filtering.

\subsection{Extended Kalman filtering (EKF)}

The non-linear state-space form as a set of first-order non-linear differential equations is given by

$$
\dot{x}=f(x)+w
$$

where $x$ is a vector of the system states, $f(x)$ is a non-linear function of those states, and $\mathrm{w}$ is a random zero-mean process. The measurement equation is considered to be a non-linear function of the states according to

$$
z=h(x)+v
$$

where $h(x)$ is a non-linear measurement matrix, $v$ is a zero-mean random process.

We assume that an approximate trajectory $x_{o}$ is available. This is referred to as the reference trajectory. The actual trajectory $x$ may then be written as

$$
x=x_{0}+\Delta x
$$

Hence, (24) and (25) become

$$
\begin{aligned}
& \dot{x_{o}}+\dot{\Delta x}=f\left(x_{o}+\Delta x\right)+w \\
& z=h\left(x_{o}+\Delta x\right)+v
\end{aligned}
$$


The Taylors series expansion yields the linearized model

$$
\begin{aligned}
& \dot{x_{o}}+\dot{\Delta x} \approx f\left(x_{o}\right)+\left[\frac{\partial f}{\partial x}\right]_{x=x_{o}} \Delta x+w \\
& z=h\left(x_{o}\right)+\left[\frac{\partial h}{\partial x}\right]_{x=x_{o}} \Delta x+v
\end{aligned}
$$

where

$$
\left.\frac{\partial f}{\partial x}\right|_{x=x_{o}}=\mathbf{F}=\left.\left[\begin{array}{l}
\frac{\partial f_{1}}{\partial x_{1}} \frac{\partial f_{1}}{\partial x_{2}} \ldots . . \\
\frac{\partial f_{2}}{\partial x_{1}} \frac{\partial f_{1}}{\partial x_{2}} \ldots \ldots . \\
\cdot
\end{array}\right] \quad \frac{\partial h}{\partial x}\right|_{x=x_{o}}=\mathbf{H}=\left[\begin{array}{l}
\frac{\partial h_{1}}{\partial x_{1}} \frac{\partial h_{1}}{\partial x_{2}} \ldots . . \\
\frac{\partial h_{2}}{\partial x_{1}} \frac{\partial h_{1}}{\partial x_{2}} \ldots . . \\
. \\
\cdot
\end{array}\right]
$$

If the reference trajectory $x_{o}$ is chosen to satisfy the differential equation

$$
\Delta \dot{x}=f\left(x_{o}\right)
$$

In view of (29) and (30), the system dynamics matrix $\Phi$ in discrete form for extended Kalman filtering becomes

$$
\Phi_{k}^{\omega}=\left[\begin{array}{ccccc}
1 & \frac{\sin (\omega T)}{\omega} & 0 & \frac{\cos (\omega T)-1}{\omega} & \omega_{x} \\
0 & \cos (\omega T) & 0 & -\sin (\omega T) & \omega_{\dot{x}} \\
0 & -\frac{\cos (\omega T)-1}{\omega} & 1 & \frac{\sin (\omega T)}{\omega} & \omega_{y} \\
0 & \sin (\omega T) & 0 & \cos (\omega T) & \omega_{\dot{y}} \\
0 & 0 & 0 & 0 & 1
\end{array}\right]
$$

Above, $\omega_{x}, \omega_{y}, .$. are given by

$$
\begin{gathered}
\omega_{x}=\frac{1}{\omega}\left[\left(T \cos \omega T-\frac{\sin \omega T}{\omega}\right) \dot{x}+\left(-T \sin \omega T-\frac{\cos \omega T-1}{\omega}\right) \dot{y}\right] \\
\left.\omega_{\dot{x}}=[-\sin \omega T) \dot{x}-\cos \omega T \dot{y}\right] T \\
\omega_{y}=\frac{1}{\omega}\left[\left(T \sin \omega T-\frac{1-\cos \omega T}{\omega}\right) \dot{x}+\left(-T \cos \omega T-\frac{\sin \omega T}{\omega}\right) \dot{y}\right]
\end{gathered}
$$




$$
\omega_{y}=[\cos \omega T \dot{x}-\sin \omega T \dot{y}] T
$$

In the extended Kalman filter operation, three measurements are considered. Referring to Figure 3 these are

$$
\begin{array}{ll}
\theta=\operatorname{arctg}(y / x)=\operatorname{arctg}\left(x_{3} / x_{1}\right) & \text { angle } \\
r=\sqrt{x^{2}+y^{2}}=\sqrt{x_{1}^{2}+x_{3}^{2}} & \text { distance } \\
v=\sqrt{v_{x}^{2}+v_{y}^{2}}=\sqrt{x_{2}^{2}+x_{4}^{2}} & \text { velocity }
\end{array}
$$

From (36), the linearized measurement matrix in terms of state variables becomes

$$
\mathbf{H}=\left[\begin{array}{ccccc}
\frac{-1}{x_{1}^{2}+x_{3}^{2}} & 0 & \frac{x_{1}}{x_{1}^{2}+x_{3}^{2}} & 0 & 0 \\
\frac{x_{1}}{\sqrt{x_{1}^{2}+x_{3}^{2}}} & 0 & \frac{x_{3}}{\sqrt{x_{1}^{2}+x_{3}^{2}}} & 0 & 0 \\
0 & \frac{x_{2}}{\sqrt{x_{2}^{2}+x_{4}^{2}}} & 0 & \frac{x_{4}}{\sqrt{x_{2}^{2}+x_{4}^{2}}} & 0
\end{array}\right]
$$

Above $x_{1}, x_{2}, x_{3}, x_{4}$ are the state variables which are defined as $x_{1}=x, x_{2}=y, x_{3}=v_{x}$, and $x_{4}=v_{y}$, respectively.

\subsection{Estimation}

In this work, the Kalman filter is an estimator of states of a dynamic system with a minimal error (innovation) variance and in this sense it is optimal. In order to explain the estimation in detail, the filter equations taking the discrete time point $k$ as reference are briefly given below. A general dynamic system given in a form

$$
\begin{gathered}
x(k+1)=A(k) x(k)+B(k) w(k) \\
z(k)=C(k) x(k)+v(k)
\end{gathered}
$$

is terminologically referred to as state-space. Above A is the system matrix; B process noise matrix; $\mathrm{C}$ is the measurement matrix. Further, $w(k)$ and $v(k)$ are Gaussian process noise and measurement noise respectively with the properties

$$
\begin{aligned}
& E\{w(k)\}=0 \\
& E\left\{w(k) w(l)^{T}\right\}=Q(k) \text { for } k=l \\
& =0 \text { otherwise } \\
& E\{v(k)\}=0 \\
& E\left\{v(k) v(l)^{T}\right\}=R(k) \text { for } k=l \\
& =0 \text { otherwise }
\end{aligned}
$$


The estimation in Kalman filter is accomplished recursively which with the notations in the literature became standard matrix equations formulation and it reads

$$
\begin{gathered}
x(k+1 \mid k)=A(k) x(k \mid k) \\
P(k+1 \mid k)=A(k) P(k \mid k) A(k)^{T}+B(k) Q(k) B(k)^{T} \\
K(k+1)=\frac{P(k+1 \mid k) C(k+1)^{T}}{C(k+1) P(k+1 \mid k) C(k+1)^{T}+R(k+1)}
\end{gathered}
$$

So that the updated as the measurements $z$ are available the updated state variables and covariance matrix are

$$
\begin{gathered}
x(k+1 \mid k+1)=x(k+1 \mid k)+K(k+1)[z(k+1)-C(k+1) x(k+1 \mid k)] \\
\boldsymbol{z}(k+1)-C(k+1) x(k+1 \mid k)=\text { innovation } \\
P(k+1 \mid k+1)=[I-K(k+1) C(k+1)] P(k+1 \mid k)
\end{gathered}
$$

$\mathrm{N}$-level multiresolutional dynamic system in a vector form can be described by

$$
\begin{aligned}
& x^{[N]}\left(k_{N}+1\right)=A^{[N]}\left(k_{N}\right) x^{[N]}\left(k_{N}\right), \\
& z^{[i]}\left(k_{i}\right)=C^{[i]}\left(k_{i}\right) x^{[i]}\left(k_{i}\right)+v^{[i]}\left(k_{i}\right) \\
& i=1, \ldots, N
\end{aligned}
$$

where $\mathrm{i}=\mathrm{N}$ is the highest resolution level, so that

$$
\begin{aligned}
& E\left[w^{[N]}\left(k_{N}\right)\right]=0, \\
& E\left[w^{[N]}\left(k_{N}\right) w^{[N]}\left(l_{N}\right)^{T}\right]=Q^{[N]}\left(k_{N}\right), \quad k=l \\
& =0 \quad k \neq l
\end{aligned}
$$

Referring to the measurements $z^{[i]}\left(k_{i}\right)$ at different resolution levels, we write

$$
\begin{aligned}
& E\left[w^{[i]}\left(k_{i}\right)\right]=0, \\
& E\left[w^{[i]}\left(k_{i}\right) w^{[i]}\left(l_{i}\right)^{T}\right]= \\
& \\
& =0 \quad Q^{[i]}\left(k_{i}\right), \quad k=l \\
& =0 \quad k \neq l
\end{aligned}
$$

and

$$
\begin{aligned}
& E\left[v^{[i]}\left(k_{i}\right)\right]=0, \\
& E\left[v^{[i]}\left(k_{i}\right) v^{[i]}\left(l_{i}\right)^{T}\right]=R^{[i]}\left(k_{i}\right), \quad k=l \\
& =0 \quad k \neq l
\end{aligned}
$$


Kalman filter is used to combine the information from the measurements at different resolutional levels and enhance the state estimation rather than to employ single measurement at each time-step.

\section{Wavelets}

\subsection{Multiresolutional decomposition}

Wavelet analysis is basically the projection of data onto a set of basis functions in order to separate different scale information. In particular, in the discrete wavelet transform (DWT) data are separated into wavelet detail coefficients (detail-scale information) and approximation coefficients (approximation-scale information) by the projection of the data onto an orthogonal dyadic basis system (Mallat 1989). In the DWT framework, a signal $f(x)$ is decomposed into approximation and detail components to form a multiresolution analysis of the signal as

$$
f(x)=\sum_{k} a_{j o, k} \phi_{j o, k}(x)+\sum_{j=j o}^{j o+J} \sum_{k} d_{j, k} \psi_{j, k}(x) \quad j_{o}, j, k \in \mathbb{Z}
$$

where $a_{j o, k}$ denote the approximation coefficient at resolution $j_{o} ; d_{j, k}$ denotes the wavelet coefficient at resolution $j ; \phi_{j 0, k}(x)$ is a scaling function; $\psi_{j, k}(x)$ is a wavelet function at resolution $j$, and $J$ is the number of decomposition levels. The coefficients are given by

$$
\begin{aligned}
& a_{j o, k}=\left\langle f(x), \phi_{j o, k}\right\rangle \\
& d_{j, k}=\left\langle f(x), \psi_{j, k}\right\rangle \quad j_{o}, j, k \in \mathbb{Z}
\end{aligned}
$$

Above $\langle$.$\rangle denotes the inner product in the space of square integrable functions L^{2}(\angle)$. Specifically, the dyadic DWT assumes the scaling functions have the property of

$$
\phi_{j o, k}(x)=2^{j o / 2} \phi\left(2^{j o} x-k\right)
$$

and the wavelet functions

$$
\psi_{j, k}(x)=2^{j / 2} \psi\left(2^{j} x-k\right)
$$

The novel feature of wavelets is that they are localized in time and frequency as to signals. This behaviour makes them convenient for the analysis of non-stationary signals. It is an elementary introduction of wavelets by introducing a scaling function, such that

$$
\phi(t)=\sqrt{2} \sum_{k} g_{k} \phi(2 t-k)
$$

A counterpart of this function is called mother wavelet function obtained from

$$
\psi(t)=\sqrt{2} \sum_{k} h_{k} \phi(2 t-k)
$$

where $l_{k}$ and $h_{k}$ are related via the equation 


$$
h_{k}=(-1)^{k} g_{1-k}
$$

The coefficients $g_{k}$ and $h_{k}$ appear in the quadrature mirror filters used to compute the wavelet transform. $\phi(t)$ and $\psi(t)$ form orthogonal functions which constitute low-pass and high-pass filters respectively which are spaces in $L_{2}(\Re)$ where inner product of functions is defined with finite energy. The orthogonal spaces satisfy the property

$$
\phi_{m, k}(t) \cup \psi(t)_{m, k}=\phi_{m+1, k}(t)
$$

where

$$
\begin{aligned}
\phi_{m, k}(t) & =2^{m / 2} \phi\left(2^{m} t-k\right) \\
\psi_{m, k}(t) & =2^{m / 2} \psi\left(2^{m} t-k\right)
\end{aligned}
$$

$m=0$ constitutes the coarsest scale. The simplest filter coefficients are known as Haar filter and given by

$$
\begin{aligned}
h_{h} & =\left[\begin{array}{ll}
h_{1} & h_{2}
\end{array}\right] \\
& =\frac{1}{\sqrt{2}}\left[\begin{array}{ll}
1 & 1
\end{array}\right] \\
g_{h} & =\left[\begin{array}{ll}
g_{1} & g_{2}
\end{array}\right] \\
& =\frac{1}{\sqrt{2}}\left[\begin{array}{ll}
1 & -1
\end{array}\right]
\end{aligned}
$$

If one sensor is used at the highest resolutional level i.e., $\mathrm{i}=3$ the measurements at different resolution levels can be obtained by the decomposition scheme shown in figure 4 .

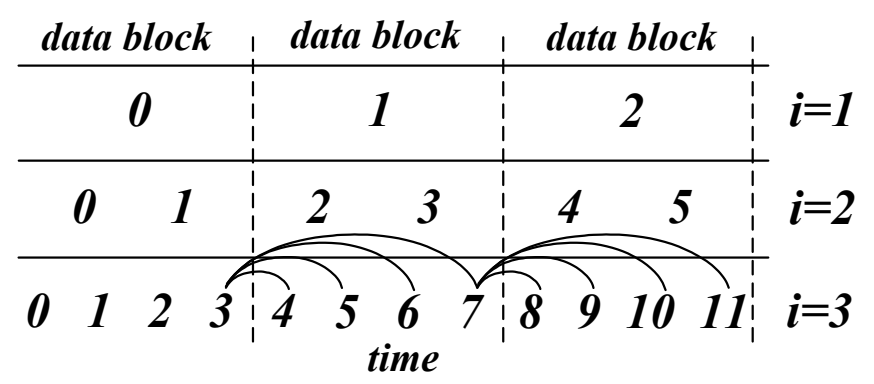

Fig. 4. Measurements at different resolution levels

In this scheme each data block at the highest resolution level (i=3) contains 4 samples. Wavelet decomposition of this block of samples is shown in figure 5 .

In figure 5, the measurements are uniform. This means measurement time points in a lower resolution are exactly at the mid of the two points of measurement times at the higher resolutional level, as indicated in figure 4 . Within a data block, the state variables at resolution level $i$ are designated as 


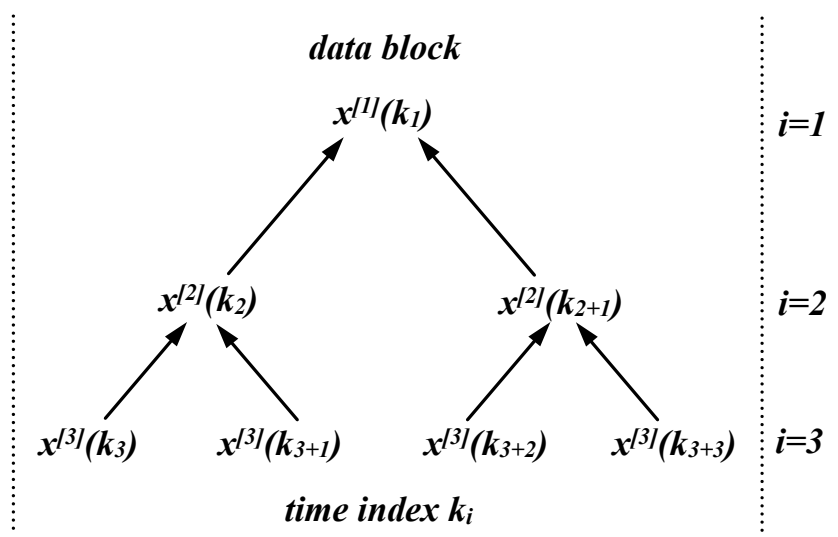

Fig. 5. Wavelet decomposition of state variables in a data block

$$
\begin{gathered}
X_{m}^{[i]}=\left[\begin{array}{l}
x^{[i]} k(i) \\
x^{[i]} k(i+1) \\
\cdots \cdots \\
x^{[i]} k\left(i+2^{i-1}\right)
\end{array}\right] \\
x_{k(i)}^{[i]}=\left[\begin{array}{c}
x_{k, 1}^{[i]} \\
x_{k, 2}^{[i]} \\
\ldots \\
x_{k, p}^{[i]}
\end{array}\right]
\end{gathered}
$$

where $m$ is data block index and $p$ is the number of state variables. In a data block, there are $2^{i-1}$ state variables. Each state variable has $p$ state components. A lower resolution state variable is computed from

$$
x^{[i]}\left(k_{i}\right)=h_{1} x^{[i+1]}\left(k_{i+1}\right)+h_{2} x^{[i+1]}\left(k_{i+1}+1\right)
$$

where $h_{1}$ and $h_{2}$ are the Haar low-pass filter coefficients. The details component i.e., high frequency part after the decomposition is computed via

$$
y^{[i]}\left(k_{i}\right)=g_{1} x^{[i+1]}\left(k_{i+1}\right)+g_{2} x^{[i+1]}\left(k_{i+1}+1\right)
$$

where $g_{1}$ and $g_{2}$ are the Haar high-pass filter coefficients. The reconstruction of the states is carried out by combining (65) and (66) in a matrix equation form as given below.

$$
\left[\begin{array}{l}
x^{[i+1]}\left(k_{i+1}\right) \\
x^{[i+1]}\left(k_{i+1}+1\right)
\end{array}\right]=\boldsymbol{h}^{* T} \boldsymbol{x}^{[i]}\left(k_{i}\right)+\boldsymbol{g}^{* \boldsymbol{T}} \boldsymbol{y}^{[i]}\left(k_{i}\right)
$$

where $h^{*}$ and $g^{*}$ are mirror filters of $h$ and $g$ counterparts; wavelet decomposition and reconstruction is carried out according to the scheme shown in figures 6 and 7. 


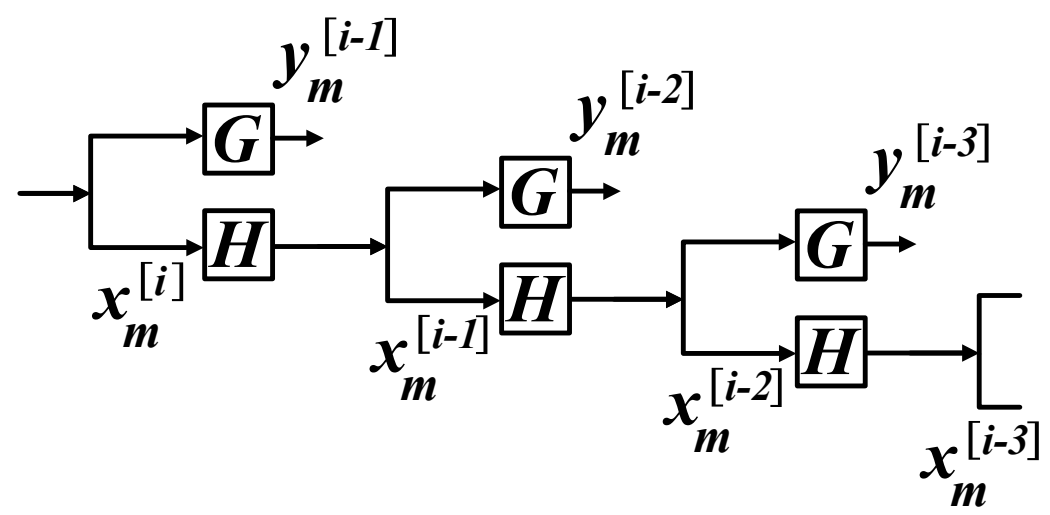

Fig. 6. Wavelet decomposition of state variables in a data block

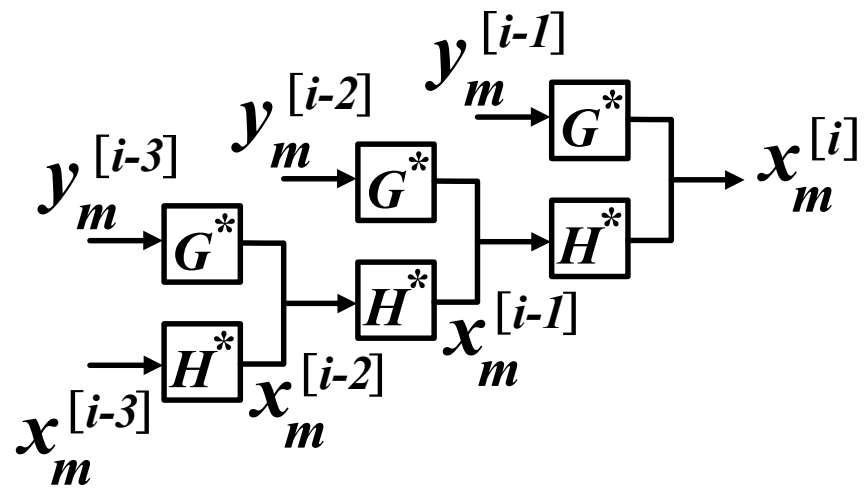

Fig. 7. Wavelet reconstruction of state variables in a data block

The wavelet matrix operator $\mathrm{G}$ and the scaling matrix operator $\mathrm{H}$ in the decomposition and their counterparts $\mathrm{G}^{*}$ and $\mathrm{H}^{*}$ in the reconstruction contain two-tap Haar filters and they related by

$$
G^{*}=G^{T} \quad H^{*}=H^{T}
$$

The operators $\mathrm{G}$ and $\mathrm{H}$ are called Quadrature Mirror Filters (QMF) for wavelet decomposition and $\mathrm{G}^{*}$ and $\mathrm{H}^{*} \mathrm{QMF}$ for reconstruction. A QMF has the following properties.

$$
\begin{aligned}
& H^{*} H+G^{*} G=1 \\
& {\left[\begin{array}{cc}
H^{*} H & H G^{*} \\
G H^{*} & G G^{*}
\end{array}\right]=\left[\begin{array}{ll}
I & 0 \\
0 & I
\end{array}\right]}
\end{aligned}
$$


where I is the identity matrix and (68) implies that filter impulse responses form an orthonormal set. It is to note that the state variable estimations are carried out at the respective resolution levels, as follows.

$$
H^{i+1 \rightarrow i}=\operatorname{diag}\left\{(1 / \sqrt{2}) h_{h}^{(1)},(1 / \sqrt{2}) h_{h}^{(2)}, \ldots .,(1 / \sqrt{2}) h_{h}^{(K)}\right\}
$$

where $h_{h}{ }^{[i]}$ is scaled two-tap Haar lowpass filters on the diagonal; $K$ is the number of filters involved in a decomposition from the resolution level $i+1$ to $i$. For instance from 2 to 1 , then $i=1$, and $K$ is given by

$$
K=2^{[i-1]}=1
$$

as this is seen in figure 5 where $2^{[i-1]}$ pairs of state variables in $X_{m}^{i+1}$ are transformed to $2^{[i-1]}$ lower resolution variables in $X_{m}{ }^{i}$. For the $p$ number of components in a state variable as seen in (64), the $H$ the scaling matrix operator is composed of $p$ number of $H^{i+1} \rightarrow^{i}$ matrices at the diagonal as

$$
H=\operatorname{diag}\left\{H_{[1]}^{i+1 \rightarrow i}, H_{[2]}^{i+1 \rightarrow i}, \ldots \ldots, H_{[p]}^{i+1 \rightarrow i}\right\}
$$

where each $H_{[i]^{i+1} \rightarrow 1}$ is given by (70). Similarly, for the reconstruction filter, we write

$$
G^{i+1 \rightarrow i}=\operatorname{diag}\left\{(\sqrt{2}) g_{h}^{(1)},(\sqrt{2}) g_{h}^{(2)}, \ldots .,(\sqrt{2}) g_{h}^{(K)}\right\}
$$

The wavelet matrix operator for $\mathrm{G}$ for the wavelet coefficients at resolution level $i$ from the resolution level $i+1$

$$
G=\operatorname{diag}\left\{G_{[1]}^{i+1 \rightarrow i}, G_{[2]}^{i+1 \rightarrow i}, \ldots \ldots, G_{[p]}^{i+1 \rightarrow i}\right\}
$$

where $K$ is given by (71). For the inverse transform scheme given by figure 7 , we write

$$
H^{*}=\operatorname{diag}\left\{H_{[1]}^{i \rightarrow i+1}, H_{[2]}^{i \rightarrow i+1}, \ldots \ldots, H_{[p]}^{i \rightarrow i+1}\right\}
$$

and

$$
G^{*}=\operatorname{diag}\left\{G_{[1]}^{i \rightarrow i+1}, G_{[2]}^{i \rightarrow i+1}, \ldots \ldots, G_{[p]}^{i \rightarrow i+1}\right\}
$$

Where each $H_{[i]^{i}}{ }^{i+1}$ and $G_{[i]^{i}}{ }^{i+1}$ is given by

$$
\begin{gathered}
H^{i \rightarrow i+1}=\operatorname{diag}\left\{(\sqrt{2}) h_{h}^{T(1)},(\sqrt{2}) h_{h}^{T(2)}, \ldots,(\sqrt{2}) h_{h}^{T(K)}\right\} \\
G^{i \rightarrow i+1}=\operatorname{diag}\left\{(1 / \sqrt{2}) g_{h}^{T(1)},(1 / \sqrt{2}) g_{h}^{T(2)}, \ldots .,(1 / \sqrt{2}) g_{h}^{T(K)}\right\}
\end{gathered}
$$

Above $T$ indicates transpose.

\subsection{Multiresolution by sensory measurements}

In subsection A wavelet decomposition is presented where N-level wavelet decomposition scheme lower level measurements are obtained basically by means of wavelet decomposition. This implies that for a state variable all measurements are obtained by a single sensor associated with that state variable. However in the present case we consider 
multiple sensors for the same state variable while the sensors are operated in different resolutions. Referring to figure 4, 3 sensors of different resolutions are considered. Since sensors are operated independently the measurements are non-uniform, in general. This means measurements in the lower resolution are not necessarily at the mid of the two points of the measurement times at the higher resolutional level. This is depicted in figure 8 . Uniform sampling is seen in figure 4.

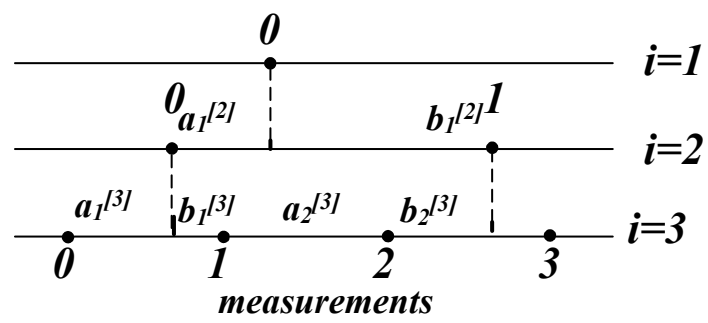

Fig. 8. Non-uniform sampling in a data block of three resolutions

Before explaining the fusion process, the wavelet decomposition for the non-uniform case will be explained in detail since this is central to this study. Decomposing the state variables at time indices 0 and 1 at resolution level $i=3$ into a single state variable at time index 0 at resolution level $i=2$ can be achieved by lowpass filter $h^{[2]}(1)$ as follows.

$$
h^{[2]}(1)=\left[\frac{b_{1}^{[3]}}{\frac{a_{1}^{[3]}}{a_{1}^{[3]}+b_{1}^{[3]}}} \frac{a_{1}^{[3]}+b_{1}^{[3]}}{]}\right.
$$

which can be written in general form

$$
h^{[i]}(1)=\left[\begin{array}{ll}
\frac{b_{k_{i}}^{[i+1]}}{a_{k_{i}}^{[i+1]}+b_{k_{i}}^{[i+1]}} & \frac{a_{1}^{[i+1]}}{a_{k_{i}}^{[i+1]}+b_{k_{i}}^{[i+1]}}
\end{array}\right]
$$

where index $i$ denotes the resolution level; $k_{i}$ is the time index at the resolution level $i ; a_{k i}{ }^{[i+1]}$ and $b_{k i}{ }^{[i+1]}$ are the relative time intervals. The lowpass filter $h^{[i]}\left(k_{i}\right)$ for deriving a coarsened estimate state variable at time index $k_{i}$ and at resolution level $i$ is based on the appropriate pair of estimated state variables at resolution level $i+1$. As the lowpass filter is determined, the highpass filter and the inverse filters can be determined by the filterbank implementation of the Quadrature Mirror Filter (QMF) shown in figures 6 and 7. Hence from lowpass filter $h^{[i]}\left(k_{i}\right)$ the highpass filter $g^{[i]} k(i)$ and the inverse filters $h_{i n v}{ }^{[i]}\left(k_{i}\right)$ and $g_{i n v}{ }^{[i]}\left(k_{i}\right)$ are determined as given below that they satisfy the constraints given by (69).

$$
\begin{gathered}
g^{[i]}\left(k_{i}\right)=\left[\frac{2 b_{k_{i}}^{[i+1]}}{a_{k_{i}}^{[i+1]}+b_{k_{i}}^{[i+1]}} \frac{-2 a_{1}^{[i+1]}}{a_{k_{i}}^{[i+1]}+b_{k_{i}}^{[i+1]}}\right] \\
h_{i n v}{ }^{[i]}\left(k_{i}\right)=\left[\frac{0.5\left(a_{k_{i}}^{[i+1]}+b_{k_{i}}^{[i+1]}\right)}{b_{k_{i}}^{[i+1]}} \frac{0.5\left(a_{k_{i}}^{[i+1]}+b_{k_{i}}^{[i+1]}\right)}{a_{k_{i}}^{[i+1]}}\right]
\end{gathered}
$$


and

$$
g_{\text {inv }}{ }^{[i]}\left(k_{i}\right)=\left[\frac{0.5\left(a_{k_{i}}{ }^{[i+1]}+b_{k_{i}}^{[i+1]}\right)}{2 b_{k_{i}}^{[i+1]}} \frac{-0.5\left(a_{k_{i}}{ }^{[i+1]}+b_{k_{i}}^{[i+1]}\right)}{2 a_{k_{i}}^{[i+1]}}\right]
$$

For $a_{k i}[i+1]=b_{k i}[i+1]$, the filters reduce to Haar filters given (61) and (62) and shown in figures 6 and 7.

In this implementation the scaling and wavelet operators $\mathrm{H}$ and $\mathrm{G}$ for decomposition $\mathrm{i}+1 \rightarrow \mathrm{i}$ are given by

$$
\begin{aligned}
& H^{i+1 \rightarrow i}=\operatorname{diag}\left\{h^{[i]}\left(k_{i}\right), h^{[i]}\left(k_{i}+1\right), \ldots, h^{[i]}\left(k_{i}+2^{i-1}-1\right)\right\} \\
& G^{i+1 \rightarrow i}=\operatorname{diag}\left\{g^{[i]}\left(k_{i}\right), g^{[i]}\left(k_{i}+1\right), \ldots, g^{[i]}\left(k_{i}+2^{i-1}-1\right)\right\}
\end{aligned}
$$

For $a_{k i}{ }^{[i+1]}=b_{k i} i^{[i+1]}(84)$ reduces to (70) and (73).

The inverse scaling and wavelet operators $\mathrm{H}$ and $\mathrm{G}$ for construction $\mathrm{i} \rightarrow \mathrm{i}+1$ are given by

$$
\begin{aligned}
& H^{i \rightarrow i+1}=\operatorname{diag}\left\{h_{i n v}{ }^{[i]}\left(k_{i}\right)^{T}, h_{i n v}{ }^{[i]}\left(k_{i}+1\right)^{T}, \ldots, h_{i n v}{ }^{[i]}\left(k_{i}+2^{i-1}-1\right)^{T}\right\} \\
& G^{i \rightarrow i+1}=\operatorname{diag}\left\{g_{\text {inv }}{ }^{[i]}\left(k_{i}\right)^{T}, g_{\text {inv }}{ }^{[i]}\left(k_{i}+1\right)^{T}, \ldots, g_{\text {inv }}{ }^{[i]}\left(k_{i}+2^{i-1}-1\right)^{T}\right\}
\end{aligned}
$$

For $a_{k i}^{[i+1]}=b_{k i}[i+1] \quad(84)$ and (85) reduces to (77) and (78).

\section{Fusion process as multiresolutinal dynamic filtering (MDF)}

The fusion of information is central to this research. Therefore, in the preceding section wavelet decomposition and reconstruction is presented in vector form for the sake of explaining the fusion process in detail. However the wavelet decomposition in this work is not used. This is simply because lower resolution level sensory measurements are obtained from associated sensors and not from wavelet decomposition of the highest resolution level sensory measurements. Therefore only the wavelet reconstruction is relevant. The lower resolution level measurements are used to update the estimated information at this very level. Afterwards, this information is transformed to higher resolution level information by inverse wavelet transform where the inverse transformation wavelet coefficients, that is the detail coefficients, are not involved in this process as they are all zero. Because of this reason the transformed information at a higher resolution level is the same as the information lying in the preceding lower level. But this very information at the higher resolution level timely coincides with the sensory information of this level. This is achieved by non-uniform formulation of wavelet transform. By doing so, the independent operation of multiresolutional sensors is aimed to make the information fusion effective. The actual implementation in this work is explicitly as follows. Referring to figure 8, a data block has four sensory measurement samples at the highest resolution $(i=3)$ and one sensory sample in the lowest resolution $(i=1)$. The resolution level between the highest and lowest contains two sensory measurements. By means of inverse wavelet transform the updated estimations at levels $i=1$ and $i=2$ are transformed to highest level separately providing the estimate of the signal of the resolution index $i=1$ and $i=2$ and the highest level $(i=3)$. In the level one, a single estimation, in the level two, two updated estimations are projected to highest level. In the 
level three the estimations are updated for four samples. At all levels the estimations are updated by Kalman filtering for $i=1,2$, and 3. Signals from different resolutional levels are projected to the highest resolution level so that they all have four samples in a data block. The basic update scheme for dynamic multiresolutional filtering is shown in Fig. 9 where at each resolutional level, when the measurement $Z$ is available, the state variables are updated and when the block $m$ is complete the inverse wavelet transform and fusion is performed. During the inverse transformation the wavelet coefficients are all zero due to nonperformed wavelet decomposition.

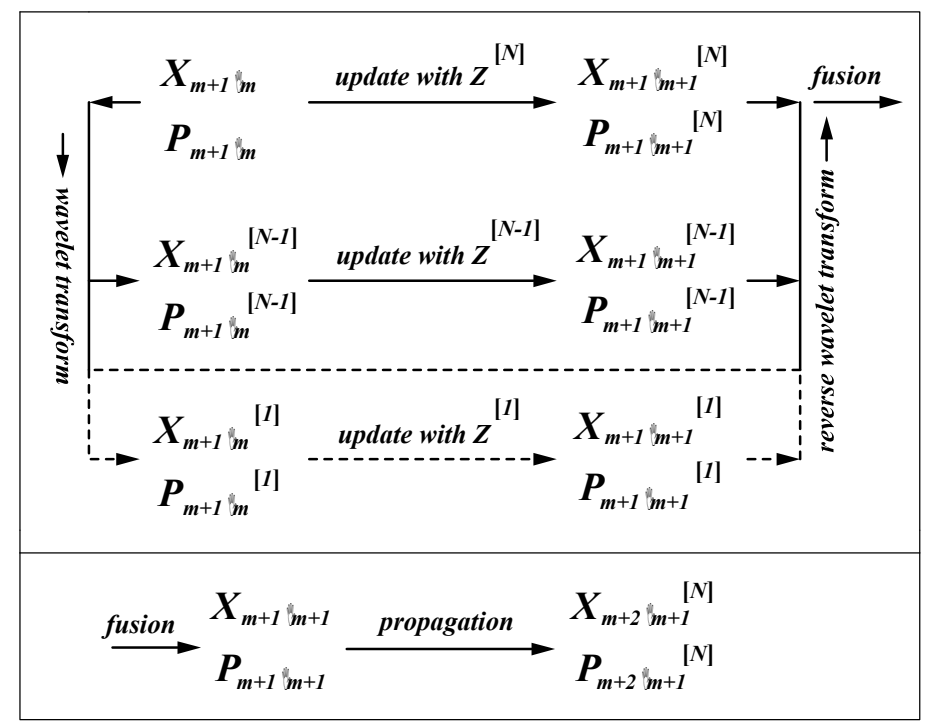

Fig. 9. Wavelet decomposition of state variables in a data block $m$

Explicitly, the basic update scheme is as follows.

$$
X_{m+1 \mid m+1}{ }^{[i]}=X_{m+1 \mid m}{ }^{[i]}+K_{m+1}{ }^{[i]}\left(Z_{m+1}{ }^{[i]}-C_{m+1}{ }^{[i]} X_{m+1 \mid m}{ }^{[i]}\right)
$$

and

$$
P_{X X_{m+1 \mid m+1}}{ }^{[i]}=\left(I-K_{m+1}{ }^{[i]} C_{m+1}{ }^{[i]}\right) P_{X X_{m+1 \mid m}}{ }^{[i]}
$$

The minimum variance Kalman gain matrix $K_{m+1}{ }^{[i]}$ at each level, is determined by

$$
K_{m+1}{ }^{[i]}=P_{X X_{m+1 \mid m}}{ }^{[i]} C_{m+1}{ }^{[i] T}\left(C_{m+1}{ }^{[i]} P_{X X_{m+1 \mid m}}{ }^{[i]} C_{m+1}{ }^{[i] T}+R_{m+1}{ }^{[i]}\right)^{-1}
$$

where the measurent matrix $C_{m=1}^{[i]}$ and $R_{m+1}^{[i]}$ are given by

$$
C_{m+1}^{[i]}=\operatorname{diag}\left[\begin{array}{ll}
C^{[i]}\left[(m+1) 2^{i-1}\right], C^{[i]}\left[(m+1) 2^{i-1}-2^{i-1}+1\right], \ldots, \\
\ldots . & , C^{[i]}\left[(m+1)+2^{i-1}+1\right]
\end{array}\right]
$$




$$
R_{m+1}^{[i]}=\operatorname{diag}\left[\begin{array}{cc}
R^{[i]}\left[(m+1) 2^{i-1}\right], R^{[i]}\left[(m+1) 2^{i-1}-2^{i-1}+1\right], \ldots, \\
\ldots . & , R^{[i]}\left[(m+1)+2^{i-1}+1\right]
\end{array}\right]
$$

Once, the sequences of updated state variables and error covariances $X_{m+1 \mid m+1}^{[N, i]}$ and $P_{m+1 \mid m+1}^{[N, i]}$ for $i=1,2, . ., N$, are determined, they must be fused to generate an optimal $X_{m+1 \mid m+1}^{[N F]}$ and $P_{m+1 \mid m+1}{ }^{[N F]}$. For the minimum fusion error covariance $P_{m+1 \mid m+1}^{[N F]}$ as derived in (Hong 1991; Hong 1992), the fused estimate $X_{m+1 \mid m+1}^{[N F]}$ is calculated as

$$
\begin{aligned}
& X_{m+1 \mid m+1}{ }^{[N F]} \\
& =P_{m+1 \mid m+1}[N F\}\left[\sum_{i=1}^{N}\left(P_{m+1 \mid m+1}^{[N, i]}\right)^{-1} X_{m+1 \mid m+1}^{[N, i]}-(N-1)\left(P_{m+1 \mid m}^{[N]}\right)^{-1} X_{m+1 \mid m}^{[N]}\right]
\end{aligned}
$$

where the minimum fusion error covariance $P_{m+1 \mid m+1}^{[N F]}$ is given by

$$
\left(P_{m+1 \mid m+1}^{[N F]}\right)^{-1}=\sum_{i=1}^{N}\left(P_{m+1 \mid m+1}^{[N, i]}\right)^{-1}-(N-1)\left(P_{m+1 \mid m}^{[N]}\right)^{-1} .
$$

The fused estimate $X_{m+1 \mid m+1}{ }^{[N F]}$ is a weighted summation of both predicted $X_{m+1 \mid m}{ }^{[N]}$ and updated $X_{m+1 \mid m+1}{ }^{[N, i]}$, for $i=1,2, . ., N$. The sum of the weight factors equal to the identity $I$. This can be seen by substitution of $P_{m+1 \mid m+1}{ }^{[N F]}$ given above into the expression of $X_{m+1 \mid m+1}{ }^{[N F]}$ in (91). With the estimations in different level of resolutions and finally fusion of the level-wise estimations for unified estimations form a multiresolutional distributed filtering (MDR).

\section{Experiments with the autonomous robot}

The computer experiments have been carried out with the simulated robot navigation. The state variables vector is given by (93) where $N=i$ to represent a general resolutional level.

$$
x_{k}^{[N]}(N)=\left[\begin{array}{c}
x_{k, 1}^{[N]} \\
x_{k, 2}^{[N]} \\
\cdots \\
x_{k, p}^{[N]}
\end{array}\right]
$$

Explicitly,

$$
x^{[N]} k(N)=[x, \dot{x}, y, \dot{y}, \omega]
$$

where $\omega$ is the angular rate and it is estimated during the move. When the robot moves in a straight line, the angular rate becomes zero and the other state variables namely, $x$ and $y$ 
coordinates and the respective velocities remain subject to estimation. The overall robot trajectory is shown in figure 10 where there are four lines plotted but they are all close to each other due to the scale involved. Broadly one can see approximately a linear trajectory followed by a curve trajectory and approximately another linear trajectory afterwards. The line marked by * sign represents the measurement of the data at the highest resolution level for $i=3$. The line marked by $\bullet$ is the estimation by sensor fusion. The line marked by + sign is the estimation by extended Kalman filtering for the data obtained from the sensor of the highest resolution level $(i=3)$. The line indicated by o sign is the reference trajectory. These lines are not explicitly seen in this figure. For explicit illustration of the experimental outcomes the same figure with different zooming ranges and the zooming powers are given in figures 11-18.

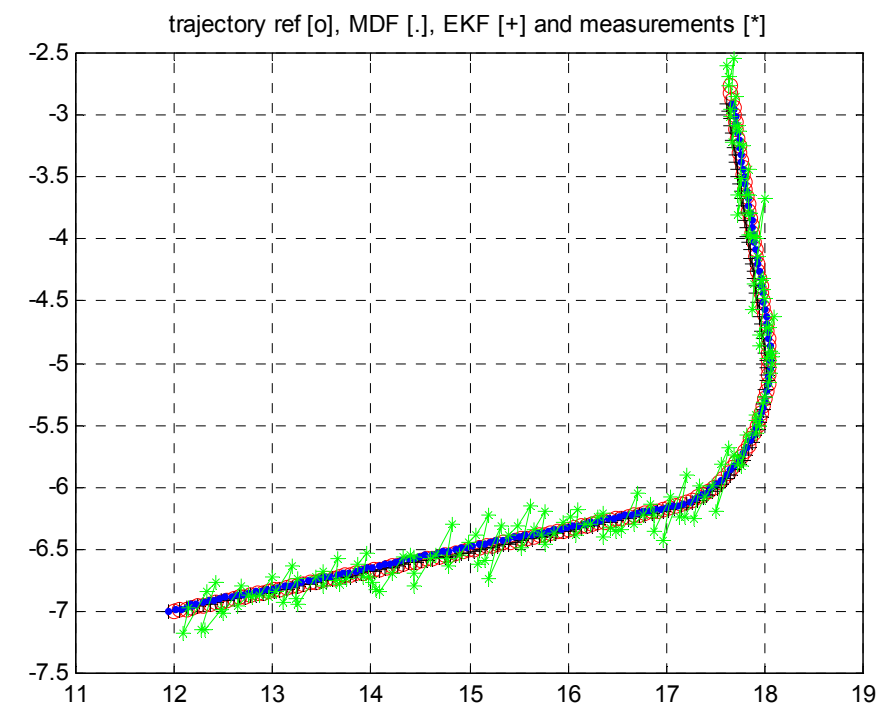

Fig. 10. The overall measurement, reference, extended Kalman filtering(EKF) and multiresolutional distributed filtering $(M D F)$ estimation of robot trajectory. The * sign is for measurement; + for $\mathrm{EKF}$; o for reference; • for MDF estimated trajectory.

Figure 11 and 12 shows the estimations in a linear mode. Figure 12 is the enlarged form of figure 11. From these figures it is seen that, the Kalman filtering is effective at the first linear part of the trajectory; namely relative to Kalman filtering estimation, the estimation by sensor fusion by MDF is inferior. In this mode the angular velocity is zero, the system matrix is linear and the linear Kalman filter is accurate enough to describe the dynamic system. During this period, the Kalman filter estimations are carried in smallest sampling time intervals. At the same period MDF estimations made in lower resolution levels are extended to the highest resolution level. However during this extension the $\mathrm{x}$ and $\mathrm{y}$ coordinates do not match exactly the estimates in the highest resolutional level because of time difference between the estimations. Explicitly, in figure 8 the estimation in the level $i=0$ is extended to estimation number 3 in the resolutional level $i=3$ where there is time difference of more than one sampling time interval. The result is higher estimation error in the MDF and this error appears to be as systematic error in estimation in the form of hangoff error, i.e., error does 


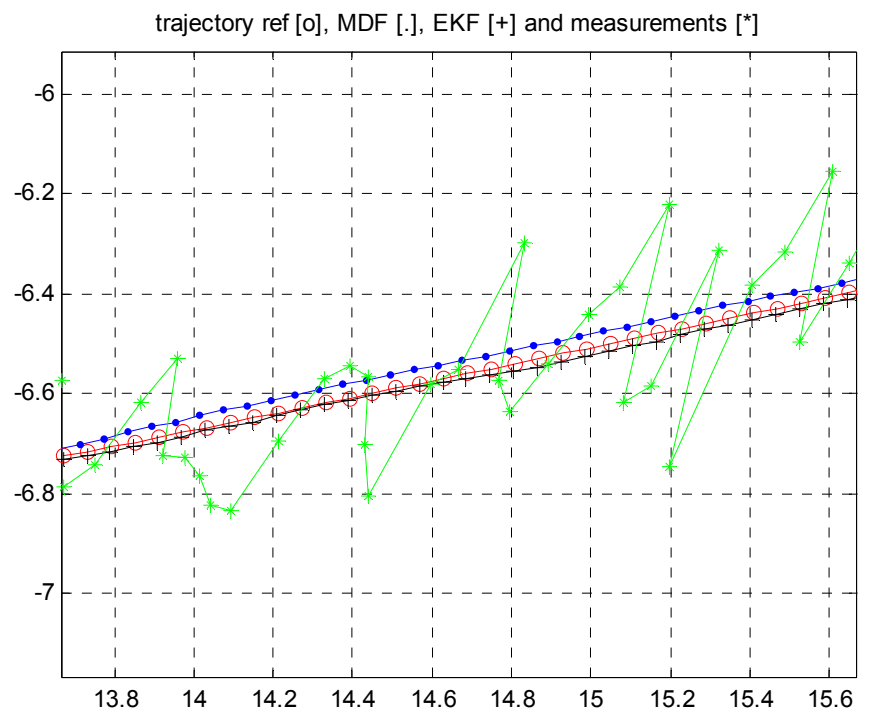

Fig. 11. Enlarged measurement, reference, extended Kalman filtering $(E K F)$ and multiresolutional distributed filtering $(M D F)$ estimation of robot trajectory in first linear period. The * sign is for measurement; + for EKF; o for reference; • for MDF estimated trajectory.

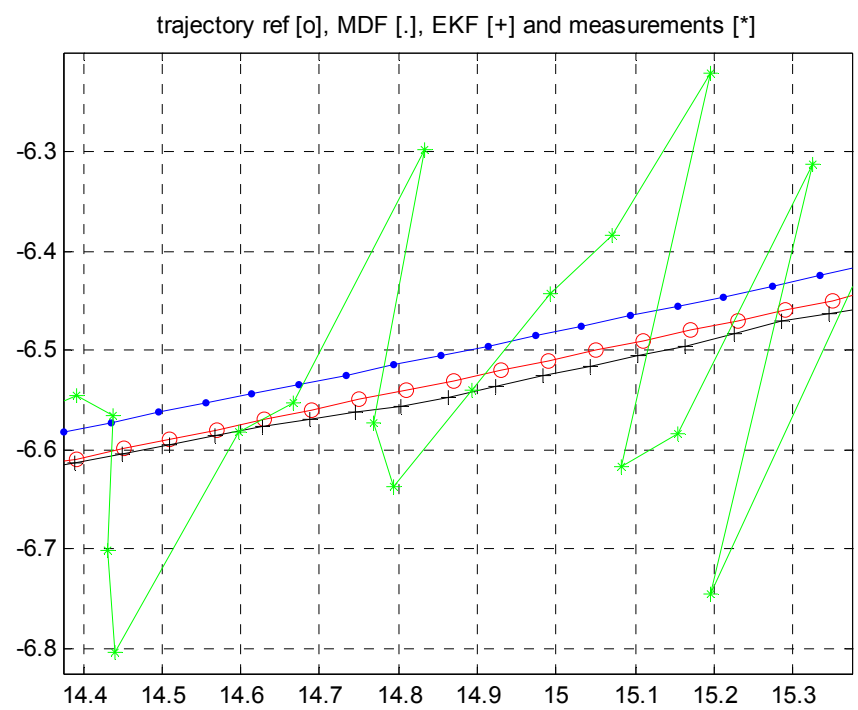

Fig. 12. Enlarged measurement, reference, extended Kalman filtering(EKF) and multiresolutional distributed filtering $(M D F)$ estimation of robot trajectory in first linear period. The * sign is for measurement; + for EKF; o for reference; • for MDF estimated trajectory. 
not go to zero. In a sensor fusion process such sensor delays are inevitable and thus delayrelated effects are inevitable. This research clearly illustrates the extent of such effects which are to be categorized eventually as errors. Consequently one can conclude that the fusion of sensors from different resolutional levels has an inherent peculiarity of latency that turns out to be undesirable outcome in some cases, as it is the case in the present situation, although this is not general as the following figures (13-18) indicate.

Figure 13 and 14 shows the estimations in a bending mode. Figure 14 is the enlarged form of figure 13. In this case estimations by sensor fusion are superior to the estimations by extended Kalman filtering. This can be explained seeing that system matrix involves the angular velocity which makes the system dynamics matrix non-linear. This results in marked separation between the reference trajectory and the estimated trajectory due to the approximation error caused by the Taylor's series expansion and ensuing linearization in the extended Kalman filtering (EKF) in the highest resolution level. One should note that the effect of this approximation error propagated four times in a data block to the time point where fusion and predictions are made for the following data block as seen in figure 4 . In this nonlinear period, sensor fusion is very effective and the difference between the estimated outcomes and the reference trajectory is apparently negligibly small. However, this is not exactly so. Because of the delay of the data from the lower resolutional levels to the highest resolutional level as described in the preceding paragraph, there is some difference between the true position and the estimated position. Nevertheless, the true trajectory is almost perfectly identified. The reason for the effectiveness in the lower resolution levels is due to more effective linearization and therefore better state estimations. Although in the lower resolutions levels error in the linearization process for EKF is greater relative to that occurred in the higher resolutional level, such modeling errors are accounted

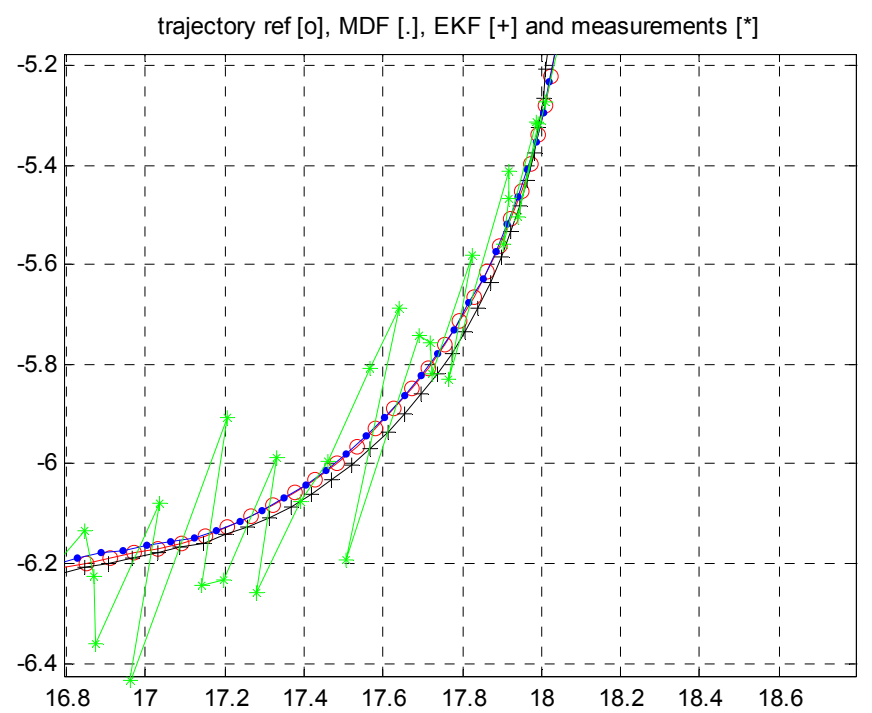

Fig. 13. Enlarged measurement, reference, extended Kalman filtering (EKF) and multiresolutional distributed filtering $(M D F)$ estimation of robot trajectory in the bending period. The * sign is for measurement; + for EKF; o for reference; • for MDF estimated trajectory. 


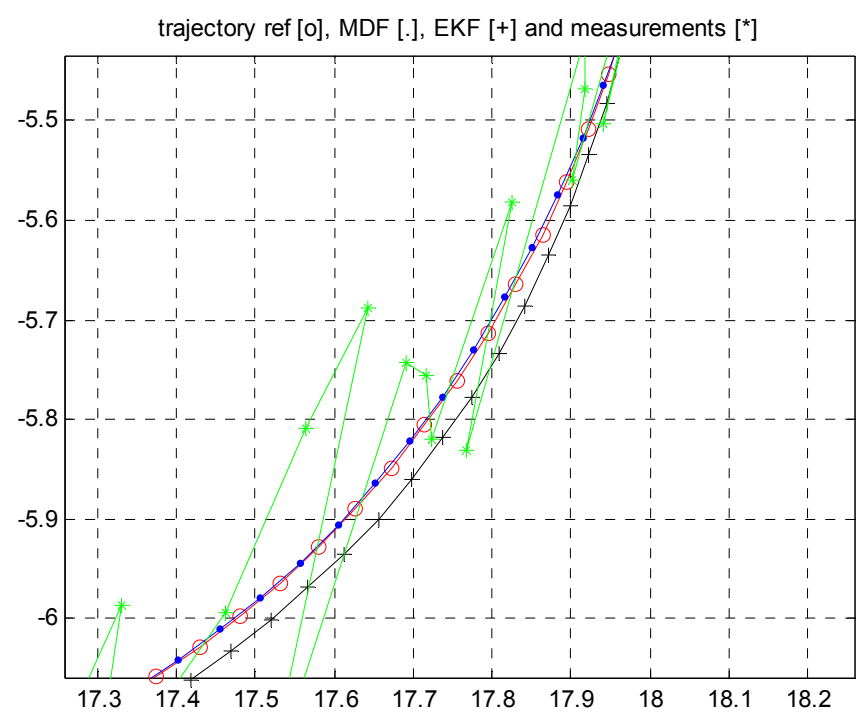

Fig. 14. Enlarged measurement, reference, extended Kalman filtering(EKF) and multiresolutional distributed filtering (MDF) estimation of robot trajectory in the bending period. The * sign is for measurement; + for EKF; o for reference; • for MDF estimated trajectory.

for in the process noise and therefore effect of errors due to linearization becomes less important. However, it should be pointed out that, in all resolutional levels, the sensor quality plays essential role on the estimation errors.

Figure 15 shows the trajectory where EKF estimation crosses the reference trajectory. This can be explained as follows. EKF estimations in the highest resolutional level without multiresolutional information, start to deviate from the reference trajectory in the bending mode as seen in figures 13 and 14. In this case Kalman filter tend to make estimations to compensate this deviation error and therefore the deviation start to become smaller. At the same time the bending information namely the angular frequency $(\omega)$ becomes effective and these two corrective joint actions in this turbulent transition period make the estimation error minimal and finally the estimated trajectory cross the reference trajectory. It is to note that in this resolutional level the Kalman filter bandwidth is relatively wide justifying the sampling rate which is the highest. As seen in (31), the system matrix is highly involved with the angular frequency and even small estimation error on $\omega$ might cause relatively high effects in this non-linear environment. After crossing, the deviations start to increase and after the bending is over it remains constant in the second linear mode in the trajectory, as seen in figures 16 and 17. On the other hand, during this period, the multiresolutional distributed filtering (MDF) estimations improve due to due to incoming bending information, the deviations become smaller and finally it crosses the reference trajectory. This crossing is shown in figure 16. The estimations at the lower resolution level are much accurate than those at the highest resolution level and by means of the sensor fusion process, the fused estimations are quite accurate at the bending mode and afterwards. This is seen in figures 13 through18. Also the effect of the information latency on the position estimation is clearly observed in these figures. 


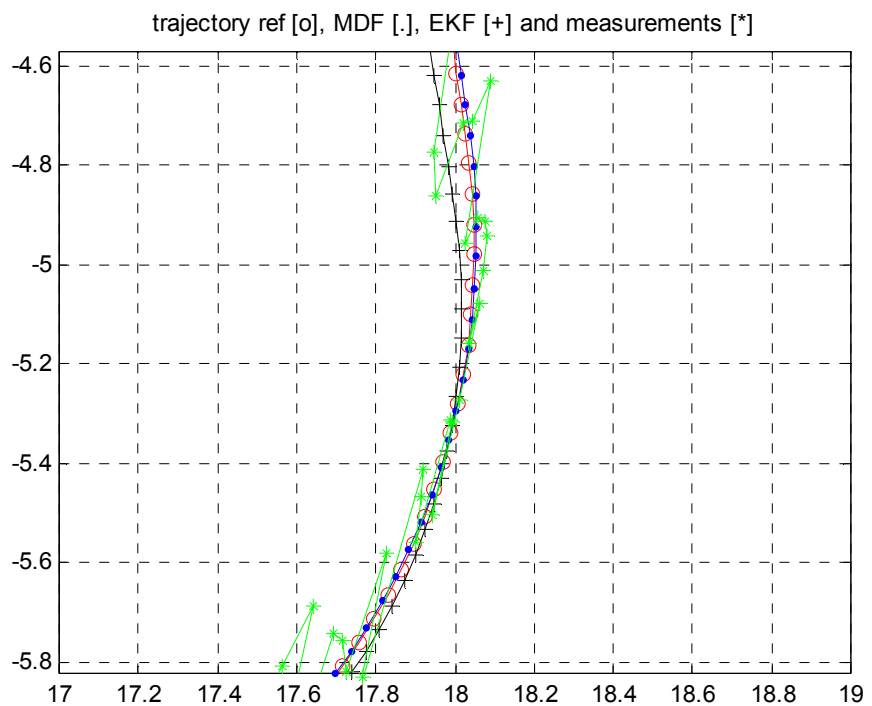

Fig. 15. Enlarged measurement, reference, extended Kalman filtering (EKF) and multiresolutional distributed filtering $(M D F)$ estimation of robot trajectory in the bending period. The * sign is for measurement; + for EKF; o for reference; • for MDF estimated trajectory.

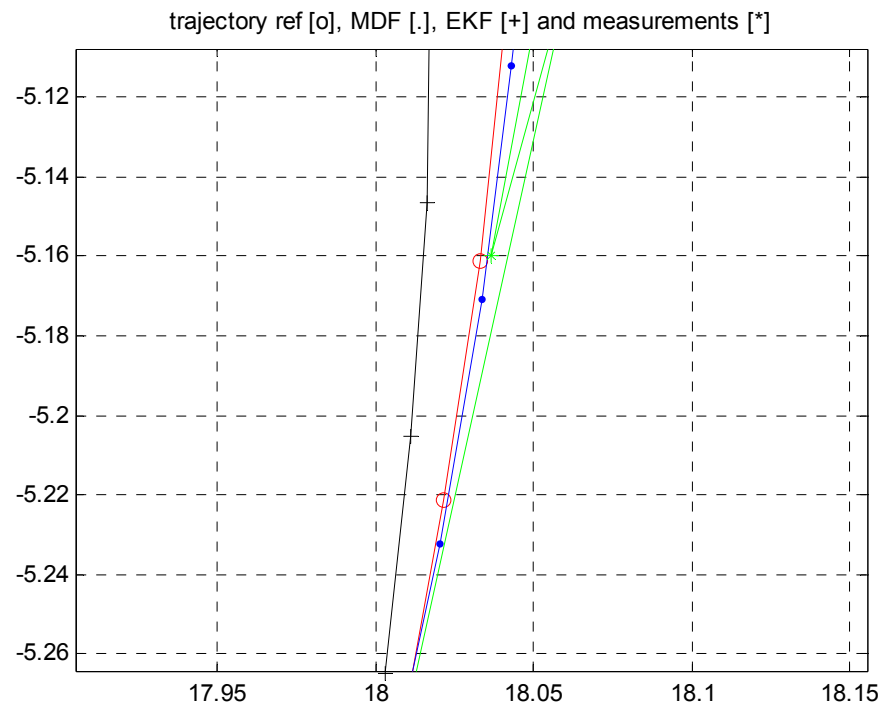

Fig. 16. Enlarged measurement, reference, extended Kalman filtering $(E K F)$ and multiresolutional distributed filtering (MDF) estimation of robot trajectory in the transition between bending and second linear periods. The * sign is for measurement; + for EKF; $\mathbf{o}$ for reference; • for $M D F$ estimated trajectory. 
Figure 17 and 18 shows the estimations in the second linear trajectory. They are the enlarged form of figure 10 at this very period. Next to satisfactory MDF estimations, the figures show the

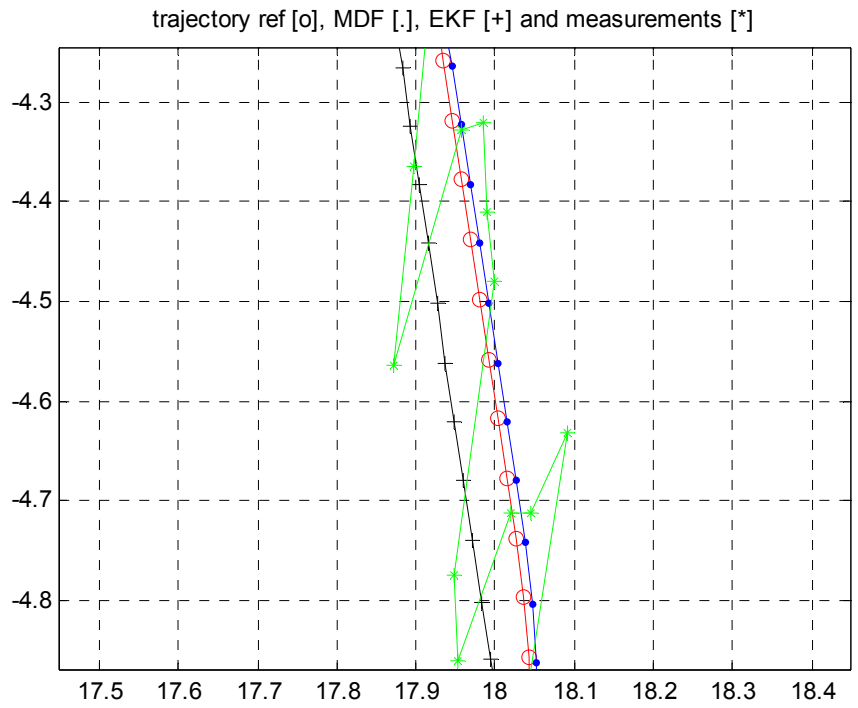

Fig. 17. Enlarged measurement, reference, extended Kalman filtering(EKF) and multiresolutional distributed filtering $(M D F)$ estimation of robot trajectory in the second linear period. The * sign is for measurement; + for EKF; o for reference; • for MDF estimated trajectory.

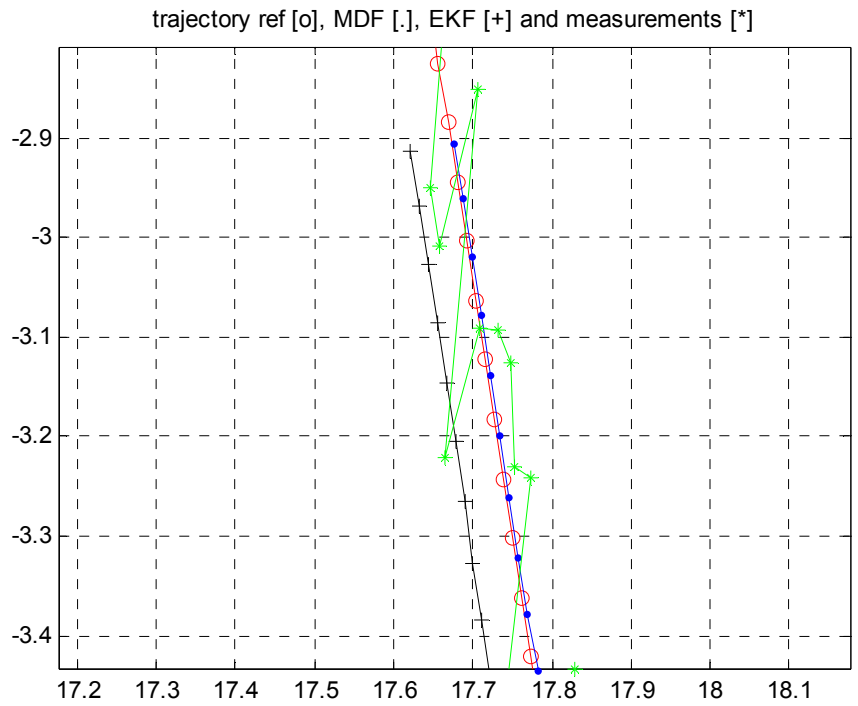

Fig. 18. Enlarged measurement, reference, extended Kalman filtering(EKF) and multiresolutional distributed filtering $(M D F)$ estimation of robot trajectory in the second linear period. The * sign is for measurement; + for EKF; o for reference; • for MDF estimated trajectory. 
estimations of the Extended Kalman filtering in the highest resolutional level. These estimations have relatively large errors which are due to the system dynamics matrix given by (31) where $\omega$ is expected to be zero in this linear period; however it is approximately zero $(\omega \approx 0)$ as calculated by Kalman filtering. The small nonzero terms in the matrix cause error in estimations which are interpreted as model errors in the Kalman filtering operation. Further such errors also cause round off errors in the covariance matrix in (43) and finally poor estimation. When the same operation is repeated by switching the matrix forcefully from bending mode to linear mode by putting $\omega=0$ in (31), the Kalman filtering estimation in the last linear period becomes comparable as illustrated in figure 11 and 12. Since the highest resolution level estimations have large errors, they have small contributions to the sensor-data fusion process and therefore the fusion results remain accurate. Figures 13 through 18 represent a firm indication of the effectiveness and robustness of the sensor fusion, in this research.

\section{Discussion}

In this work the effectiveness of multisensor-based multiresolutional fusion is investigated by means of estimation errors of mobile robot position determination. The comparison is made offline but not real-time. By doing so, a clear view presented about at what conditions the multiresolutional multi-sensor fusion process is effective and also in which circumstances the fusion process may have shortcomings and why. However, the implementation can be carried on in real-time in the form of one block ahead prediction forming the data-sensor fusion, and one step-ahead prediction at the highest resolutional level i.e., for $i=3$ without fusion process. These are illustrated in figure 4 . In both cases, i.e., real-time and off-line operations, the merits of the multiresolutional multisensor fusion remains robust although some unfavorable deviation from the existing results in real-time may occur due to a block prediction compared to 1-step-ahead prediction, obviously. Investigations on real-time operation for the assessment of the robustness are interesting since the mobile robot is especially meant for this type of operation.

\section{Conclusions}

Autonomous mobile robot navigation is a challenging issue where robot should be provided with accurate and reliable position information. Although reliable information can be provided by adding redundant sensors, the enhanced accuracy and precision information can be provided by synergistically coordinating the information from these sensors. In this respect, the present research introduced a novel information fusion concept by inverse wavelet transform using independent multiresolutional sensors. In the linear system description, the highest resolutional sensor provides enough information for optimum information processing by Kalman filtering where residual variance is minimum so that the information delivered by multiresolutional sensors can be redundant depending on the sensors's qualities and associated noises. In situations where system dynamics is non-linear, Kalman filter is still optimal in its extended formulation. However, the estimation errors in this case are dependent on the degree of the non-linearity of the system dynamics. The multiresolutional sensor fusion becomes quite effective in the non-linear case since the partial nonlinearity information of the system in different resolutional scales is available. Sensor quality is always an important factor playing role on the estimation. These features are demonstrated and the fusion process presented can easily be extended to consider realtime operation as well as some cases of probabilistic nature such as missing measurements, sensor failures and other probabilistic occurrences. 


\section{References}

Abidi, M. A. and R. C. Gonzales (1992). Multi-sensor Image Fusion. Data Fusion in Robotics and Machine Intelligence. R. S. Blum and Z. Liu, Academic Press.

Beetz, M., T. Arbuckle, et al. (2001). Integrated, Plan-based Control of Autonomous Robots in Human Environments. IEEE Intelligent Systems 16(5): 56-65.

Bellotto, N. and H. Hu (2009). Multisensor-based Human Detection and Tracking for Mobile Service Robots. IEEE Trans. System, Man, and Cybernetics-B 39(1): 167-181.

Brown, R. G. (1983). Introduction to Random Signal Analysis and Kalman Filtering. New York, John Wiley \& Sons.

Ciftcioglu, Ö. (2008). Multiresolutional Filter Application for Spatial Information Fusion in Robot Navigation. In: Advances in Robotics, Automation and Control, 355-372, J. Aramburo and A. R. Trevino (Eds.), ISBN 78-953-7619-16-9, I-Tech Publishing, Vienna, Austria.

Ciftcioglu, Ö. (2008). Shaping the Perceptual Robot Vision and Multiresolutional Kalman Filtering Implementation. Int. Journal Factory Automation, Robotics and Soft Computing(3): 62-75, ISSN 1828-6984, International Spociety for Advanced Research, www.internationalsar.org.

Ciftcioglu, Ö., M. S. Bittermann, et al. (2007). Visual Perception Theory Underlying Perceptual Navigation. Emerging Technologies, Robotics and Control Systems International Society for Advanced Research: 139-153.

Gelb, A. (1974). Applied Optrimal Estimation. Cambridge, MA, MIT Press.

Grewal, M. S. and A. P. Andrews (2001). Kalman Filtering Theory and Practice Using MATLAB. New York, Wiley.

Hong, L. (1991). Adaptive Distributed Filtering in Multi-coordinated Systems. IEEE Trans. on Aerospace and Electronic Systems 27(4): 10.

Hong, L. (1992). Distributed Filtering Using Set Models. IEEE Trans. on Aerospace and Electronic Systems 28(4): 10.

Hong, L. (1993). Multiresolutional Filtering using Wavelet Transform. IEEE Trans. on Aerospace and Electronic Systems 29(4): 1244-1251.

Hsin, H. C. and A. C. Li (2006). Wavelet-based Kalman Filtering in Scale Space for Image Fusion. Pattern Recognition and Computer Vision. C. H. C. a. P. S. P. Wang. Singapore, World Scientific.

Jazwinski, A. H. (1970). Stochastic Processes and Filtering Theory. New York Academic Press.

Kailath, T. (1981). Lectures on Wiener and Kalman Filtering. New York, Springer Verlag.

Mallat, S. G. (1989). A theory for multiresolution signal decomposition:the wavelet representation. IEEE Trans. on Pattern Analysis and Machine Intelligence. 11(7): 674-693.

Maybeck, P.S. (1982). Stochastic Models, Estimation and Control, Vol II. New York, Academic Press.

McKendall, R. and M. Mintz (1992). Data Fusion Techniques using Robust Statistics. Data Fusion in Robotics and Machine Intelligence Academic Press: 211-244.

Mendel, J. M. (1987). Kalman Filtering and Digital Estimation Techniques. New York, IEEE.

Oriolio, G., G. Ulivi, et al. (1998). Real-time Map Building and Navigation for autonomous robots in unknown environments. IEEE Trans. on Systems, Man and Cybernetics Part B: Cybernetics 28(3): 316-333.

Richardson, J. M. and K. A. Marsh (1988). Fusion of Multi-Sensor Data. Int. J. Robotics Research 7(6): 78-96.

Simon, D. (2006). Optimal State Estimation. New Jersey, Wiley Interscience.

Sorenson, H. W. (1985). Kalman Filtering: Theory and Application. New York, IEEE Press.

Wang, M. and J. N. K. Liu (2004). Online Path Searching for Autonomous Robot Navigation. IEEE Conf. on Robotics, Automation and Mechatronics, 1-3 December, Singapore: 746751, vol.2, ISBN 0-7803-8645-0 


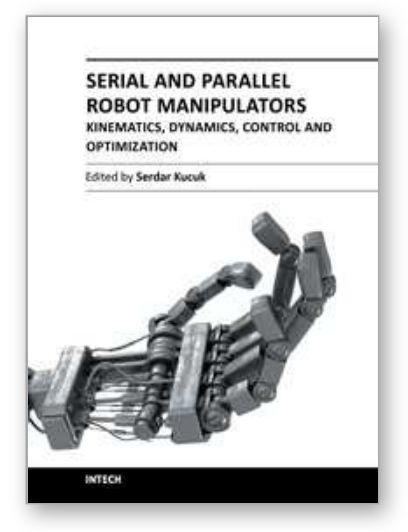

\section{Serial and Parallel Robot Manipulators - Kinematics, Dynamics, Control and Optimization}

Edited by Dr. Serdar Kucuk

ISBN 978-953-51-0437-7

Hard cover, 458 pages

Publisher InTech

Published online 30, March, 2012

Published in print edition March, 2012

The robotics is an important part of modern engineering and is related to a group of branches such as electric \& electronics, computer, mathematics and mechanism design. The interest in robotics has been steadily increasing during the last decades. This concern has directly impacted the development of the novel theoretical research areas and products. This new book provides information about fundamental topics of serial and parallel manipulators such as kinematics \& dynamics modeling, optimization, control algorithms and design strategies. I would like to thank all authors who have contributed the book chapters with their valuable novel ideas and current developments.

\section{How to reference}

In order to correctly reference this scholarly work, feel free to copy and paste the following:

Özer Çiftçioğlu and Sevil Sariyildiz (2012). Data Sensor Fusion for Autonomous Robotics, Serial and Parallel Robot Manipulators - Kinematics, Dynamics, Control and Optimization, Dr. Serdar Kucuk (Ed.), ISBN: 978-95351-0437-7, InTech, Available from: http://www.intechopen.com/books/serial-and-parallel-robot-manipulatorskinematics-dynamics-control-and-optimization/data-sensor-fusion-for-autonomous-robotics

\section{INTECH}

open science | open minds

\section{InTech Europe}

University Campus STeP Ri

Slavka Krautzeka 83/A

51000 Rijeka, Croatia

Phone: +385 (51) 770447

Fax: +385 (51) 686166

www.intechopen.com

\section{InTech China}

Unit 405, Office Block, Hotel Equatorial Shanghai

No.65, Yan An Road (West), Shanghai, 200040, China

中国上海市延安西路65号上海国际贵都大饭店办公楼405单元

Phone: +86-21-62489820

Fax: +86-21-62489821 
(C) 2012 The Author(s). Licensee IntechOpen. This is an open access article distributed under the terms of the Creative Commons Attribution 3.0 License, which permits unrestricted use, distribution, and reproduction in any medium, provided the original work is properly cited. 